\title{
BRIEF
}

\section{Interprofessional Medication Error Disclosure Training Utilizing a Telehealth Consultation Simulation}

\author{
Angela Baalmann, PharmD, ${ }^{\mathrm{a}}$ Ashley Crowl, PharmD, ${ }^{\mathrm{a}}$ Candice Coffey, MD, ${ }^{\mathrm{b}}$ Stephen Jernigan, $\mathrm{PhD},{ }^{\mathrm{c}} \mathrm{Jessica}$ \\ Kalender-Rich, MD, ${ }^{\mathrm{b}}$ Dory Sabata, ${ }^{\mathrm{c}}$ Sarah Shrader, PharmD, ${ }^{\mathrm{a}}$ Laura Zahner, ${ }^{\mathrm{d}}$ Crystal Burkhardt, PharmD ${ }^{\mathrm{a}}$ \\ ${ }^{a}$ University of Kansas, School of Pharmacy, Lawrence, Kansas \\ ${ }^{\mathrm{b}}$ University of Kansas, School of Medicine, Kansas City, Kansas \\ ${ }^{\mathrm{c}}$ University of Kansas, School of Health Professions, Kansas City, Kansas \\ ${ }^{\mathrm{d}}$ University of Kansas, Landon Center on Aging, Kansas City, Kansas
}

Corresponding Author: Ashley Crowl, University of Kansas, School of Pharmacy, 3901 Rainbow Blvd, MS 4047, Kansas City, KS 66160. Tel: 785-864-3591. Email: ancrowl@ku.edu

Submitted July 2, 2021; accepted February 18, 2022; ePublished February 2022

Objective. Health profession students must develop collaborative skills to disclose errors effectively and improve patient safety. We proposed that an interprofessional (IP) simulation utilizing telehealth technology would provide medical and pharmacy students the opportunity to practice, develop, and grow in their confidence and skills of working collaboratively and disclosing medication errors.

Methods. A three-phase IP student simulation was developed. Phase 1 included individual student preparation. An IP telehealth consultation encounter occurred in phase 2 for the error disclosure between the pharmacy and medical students. Phase 3 included faculty-led IP debrief sessions. A pre- and post-simulation survey assessed students' experiences regarding their confidence in error disclosure, use of telehealth technology, and the role of the community pharmacist. Faculty evaluated pharmacy student performance utilizing a 12-point rubric.

Results. Pre-simulation survey responses $(n=173)$ were compared to post-simulation survey responses $(n=140)$. Significant changes were seen for all students' confidence in error disclosure and use of telehealth technology. No significant change was noted in the students' understanding of the community pharmacists' role on the IP team. Pharmacy student performance-based rubric data $(n=148)$ revealed a median score of $7 / 12$ for error disclosure and IP communication items.

Conclusion. Medical and pharmacy students perceived confidence improved in IP error disclosure and use of telehealth consultation technology through this IP simulation. Pharmacy students' error disclosure and IP communication skill development were assessed through this simulation.

Keywords: interprofessional education, simulation, error disclosure, telehealth consultation, community pharmacy

\section{INTRODUCTION}

Medical errors, defined as "the failure of a planned action to be completed as intended or the use of a wrong plan to achieve an aim," often result in preventable adverse events such as morbidity and mortality. Medication errors are one of the most common types of error and frequently occur due to inadequate medication reconciliation during transitions of care. ${ }^{1,2}$ Pharmacist-led medication reconciliation has reduced health-system utilization and improved medication safety. ${ }^{2}$ Dillion and colleagues demonstrated that teams with a pharmacist showed a significant reduction in the severity of medication errors. ${ }^{3}$

It is vital that a coordinated error communication approach is utilized between health professionals, particularly when pharmacists identify medication errors during medication reconciliation. Mazan and colleagues reported that full medication error disclosures to the patient are not being achieved by practicing pharmacists and they identified the importance of integrating this skill into the pharmacy curriculum. ${ }^{4}$ Pharmacy school curricula are required to prepare and assess students' practice-readiness to collaborate in an interprofessional (IP) team to promote the health of populations through minimizing medication errors. ${ }^{5,6,7}$ To accomplish this, students must be able to display effective IP team error disclosure behaviors which include three general stages of team disclosure 1) discussion of medical error, 2) planning of disclosure to a patient, and 3) conducting an actual disclosure to the patient. ${ }^{8}$ Studies by Gardener, Krumwiede, and colleagues noted the importance of effective error disclosure communication in the IP setting while emphasizing the limited formal training graduates of health professions receive in error disclosure. ${ }^{9,10}$ Their approaches, with work from McDonough, Marshall, and colleagues, demonstrated changes in student attitudes and confidence through IP error 
disclosure simulations with standardized patient actors (SPAs) and debriefing sessions. ${ }^{9-12}$ Ragucci and colleagues conducted similar research that demonstrated improved confidence and team proficiency in disclosing medical errors. ${ }^{13}$ These studies, with research by Porier and colleagues, demonstrated increased student confidence and value in disclosing medical errors in the IP setting through the training experience. ${ }^{10,13,14}$ While evaluation of the team as a whole has been demonstrated, individual student evaluation of skill development in error disclosure is lacking. ${ }^{13}$

IP collaboration now encompasses technology such as telehealth, or the use of electronic information and telecommunication strategies, including synchronous video conferencing, to provide virtual health care to patients in a real-time, face-to-face manner. ${ }^{15,16}$ Telehealth consultation is the use of telehealth resources to facilitate collaboration and communication among health professional team members to manage mutual patients' health and jointly develop care plans. ${ }^{17}$ As students prepare for direct patient care, they must be familiar with how to properly use and collaborate with others through this format.

The purpose of this study was to understand the effect a simulation, using telehealth consultation between a medical student and pharmacy student to disclose medication errors, had on their confidence and faculty assessed competence of their skills. The primary outcomes measured were students' self-perceptions of confidence in error disclosure, use of telehealth consultation, and roles of the community pharmacist when providing medication therapy management (MTM). The secondary outcome was assessment of pharmacy students' skills in disclosing errors to another health professional.

\section{METHODS}

This simulation paired pharmacy students with medical students located on different campuses utilizing Zoom ${ }^{\circledR}$ (Version: 5.5.5) video recording software. During the 2018-19 academic year, second-year pharmacy students and thirdyear medical students were involved in the simulation experience, fulfilling both programs' IPE requirements. The simulation case timeline spanned the day of hospital discharge to the day after discharge in the community pharmacy. The simulation included three phases as detailed in Figure 1.

The first phase consisted of pre-learning activities to prepare all students for the simulation. All students reviewed a medication error identification and disclosure podcast. ${ }^{11}$ Additionally, medical students participated in a team planning simulation at the time of hospital discharge. ${ }^{18}$ All pharmacy students viewed a pre-recorded SPA encounter video of a community pharmacist providing medication reconciliation services the day after discharge. Pharmacy students were instructed to reconcile and identify medication errors based on the health information provided related to the patient's discharge and recorded video.

The second phase, the simulation encounter, consisted of a telehealth consultation between a pharmacy student as the community pharmacist and a medical student as the discharging hospital physician. During this encounter, the pharmacy student discussed concerns regarding the patient's discharge medication orders with the medical student who was responsible for the patient's discharge. Discussion between students involved rectification of discharge medication orders, addressing identified medication errors, and implementing a plan to disclose and correct the errors. Video feed of the encounter was observed by IP faculty during the encounter and for later review by pharmacy faculty via recording capabilities.

The final phase was the debrief phase of the experience. An IP faculty-conducted, structured team debrief regarding students' IP experiences occurred immediately following the simulation. Pharmacy students later participated in a pharmacy only faculty-led debrief and were able to review their individualized feedback via a performance-based rubric. The performance-based rubric was developed by pharmacy faculty and incorporated elements from the Accreditation Council for Pharmacy Education (ACPE) Standards, Center for the Advancement of Pharmacy Education (CAPE) Outcomes and core Entrustable Professional Activities (EPAs), and work of Kim and colleagues. ${ }^{5-8}$ The rubric focused on the population health promoter and interprofessional team member EPA domains and CAPE domains 2 and 3. Faculty evaluated students' pre-APPE achievement level regarding medical error disclosure and IP communication EPAs on twelve items each assigned a point-based score. Higher scores denoted higher proficiency of completion and satisfactory demonstration for each item.

Students were requested to complete a voluntary, anonymous, non-validated, twelve-question survey before and after the simulation which utilized a five-point Likert scale. Survey item development was informed by the modified Team-Oriented Medical Error Communication Assessment Tool and the faculty team. ${ }^{8}$ Of the 12 questions, there were nine shared questions designed to identify changes in student responses from the simulation in the areas of error disclosure conducted between health professionals, the use of telehealth consultation, and the roles of the community pharmacist when performing MTM. Three questions regarding the student's perception of the telehealth consultation were asked only post-simulation, and not included in this report. 
Descriptive statistical analyses were conducted for quantitative survey and rubric data using Microsoft Excel (version 15). Wilcoxon Rank Sum statistical analysis was performed on the nine survey question results using SPSS (version 22). P-values $<0.05$ were considered statistically significant. This study was approved by the University of Kansas institutional review board (\#STUDY00140041).

\section{RESULTS}

During the 2018-2019 academic year, 145 pharmacy students and 137 medicine students participated in this simulation. Due to class size differences, the eight additional pharmacy students were paired with a medicine resident or fellow. For the pre-simulation survey, 99 pharmacy responses (68\%) and 74 medical responses (54\%) were collected. For the post-simulation survey, 94 pharmacy responses (65\%) and 46 medical responses (34\%) were collected. School of medicine and pharmacy quantitative survey response data were analyzed separately, and results are provided in Table 1.

Significant improvements in perceived confidence to disclose medication errors and use telemedicine consultative services were seen for all students from pre-to-post simulation. Significant improvements were also seen in students reported understanding of MTM as performed by community pharmacists for all students. Students reported no change in their understanding of the role of the community pharmacist in patient care, both in the community setting and as a member of the IP team. Faculty evaluated all participating pharmacy student's error disclosure and IP communication skills using the performance-based rubric $(n=145)$ with results provided in Table 2. Data revealed a median overall score of 7/12 on the error disclosure and IP communication items. Students scored the highest, indicating greater competency, in the areas of communication and contributing to team discussion of the errors. The lowest scoring areas, indicating lower competency, included acknowledging that errors occurred, planning roles for disclosure of the error to the patient, and planning and anticipating patient questions.

\section{DISCUSSION}

This simulation achieved the intended learning outcomes of improving all students' perceived confidence in disclosing medication-related errors. These findings add to the prior body of evidence demonstrating benefit in developing error disclosure skills through use of IP simulations. ${ }^{9-14}$ These skills are essential to ensure health professional students are equipped to prevent future errors across health care systems as they enter practice.

Students also reported gaining confidence in using telehealth consultative services through this simulation. With the growing use of telehealth technology in health care, our simulation allowed students to gain confidence with its use before entering practice. Incorporating telehealth technology in education is noted in emerging proposals for EPAs within medical and nursing curricula but is not yet within pharmacy curricula. ${ }^{19-21}$ To our knowledge, this is the first report of combining an error disclosure simulation with this key medium of interprofessional communication to inform future practice. The use of video communication technology during the simulation allowed for multiple campuses to connect in real-time, reducing the need for travel while also allowing for the continuation of this simulation as a fully remote experience during the COVID-19 pandemic.

A significant change was noted in pre- to post- simulation survey results regarding the pharmacists' role in MTM. This is contrasted with no significant change in outcomes related to the primary role of the pharmacist in the community setting and as a member of the IP team. The absence of change noted is attributed to students likely having already developed an accurate picture of the pharmacist as a member of the IP team and their expanded role beyond medication dispensing, but not yet familiar with community pharmacists providing MTM services.

Additionally, the simulation was effective in allowing pharmacy students to demonstrate foundational skills associated with error disclosure. Currently, this simulation is the only pre-APPE learning activity where students practice error disclosure process skills within the KU pharmacy curriculum. Using the performance-based rubric median score from the 2018-2019 academic year identified students who performed below average compared to their peers, thus allowing faculty to discuss with these students individually the areas/skills they needed improvement in. Continued exposure to the skills that had the lowest performance, which were: 1) acknowledging errors occurred, 2) planning roles for disclosure to the patient, and 3) planning and anticipating the patient's response, questions, and reactions to the error disclosure, will be considered in future curricular activities. Additional data from surveys and rubric assessments collected in future simulations may confirm or refute data collected and analyzed from the 2018-2019 academic year. This IPE activity is mapped to ACPE standards, CAPE outcomes, and EPA core competencies related to IP patient care and medication error minimization. ${ }^{5-7}$ While error disclosure simulations have been studied for pharmacy students' selfreported attitudes and perceived confidence, this is the first study to specifically evaluate their skill competency in error disclosure. ${ }^{11-13}$

Limitations noted which hinder the generalizability of the findings include differing pre-and-post simulation response sizes between both medicine and pharmacy learners. The use of 'comfort' versus 'confidence' in select survey 
items may have led to a higher agreement with the statement without fully reflecting true confidence in the targeted skill. Modifications to these survey items would be beneficial to implement in future iterations of this simulation. Finally, data from only a single academic year and institution were included. Further expansion of data collection would strengthen these findings.

This study was not designed to assess the competency of the team (as a whole) in the error disclosure process as completed by Marshall, Kim, and colleagues, which made it difficult for pharmacy evaluators to assess only pharmacy students' role within the team unit's discussion surrounding the error. ${ }^{8,12}$ Additionally, this study addressed the initial steps of team discussion of the error and planning for the disclosure. Future expansion of the simulation experience may include incorporating a simulation component allowing students to disclose the error(s) identified to a SPA to build confidence and competence with this IP skill.

\section{CONCLUSION}

This IP simulation allowed students to practice utilizing IP communication to disclose a medication error, resulting in increased perceived student confidence in error disclosure. The use of telehealth consultation provided a unique educational experience and allowed multiple campuses to be involved.

\section{ACKNOWLEDGEMENTS}

This study was supported by the Kansas Reynold's Program in Aging Innovations in Interprofessional Education Grant. Simulation logistics were supported by the Neis Clinical Skills Laboratory staff at the University of Kansas Medical Center.

\section{REFERENCES}

1. Institute of Medicine (US) Committee on Quality of Health Care in America, Kohn LT, Corrigan JM, Donaldson MS, eds. To Err is Human: Building a Safer Health System. Washington (DC): National Academies Press (US); 2000.

2. Mekonnen AB, McLachlan AJ, Brien JA. Effectiveness of pharmacist-led medication reconciliation programmes on clinical outcomes at hospital transitions: a systematic review and meta-analysis. BMJ Open.

2016;6(2):e010003. Published 2016 Feb 23. doi:10.1136/bmjopen-2015-010003

3. Dillon C, Lynch G, Dean J, Purvis C, Becket L. Impact of pharmacist involvement on medication safety in interprofessional transfer of care activity. N Z Med J. 30 Jul 2021;134(1539):9-20. PMID: 34320611

4. Mazan, J. L., Lee, M. K., \& Quiñones-Boex, A. C. American pharmacists attitudes and behaviors regarding medication error disclosure. Innovations in pharmacy. 2020;11(4). doi: https://doi.org/10.24926/iip.v11i4.3373

5. Accreditation Council for Pharmacy Education. Accreditation standards and key elements for the professional program in pharmacy leading to the doctor of pharmacy degree. Standards 2016. https://www.acpeaccredit.org/pdf/Standards2016FINAL.pdf. Accessed June 24, 2021.

6. Medina MS, Plaza CM, Stowe CD, et al. Center for the advancement of pharmacy education 2013 educational outcomes. Am J Pharm Educ. 2013;77(8):162. doi:10.5688/ajpe778162

7. Haines ST, Pittenger AL, Stolte SK, et al. Core entrustable professional activities for new pharmacy graduates. Am J Pharm Educ. 2017;81(1):S2. doi:10.5688/ajpe811S2

8. Kim S, Brock D, Prouty CD, et al. A web-based team-oriented medical error communication assessment tool: development, preliminary reliability, validity, and user ratings. Teach Learn Med. 2011;23(1):68-77. doi:10.1080/10401334.2011.536896

9. Gardner AK, Lim G, Minard CG, Guffey D, Pillow MT. A cross-specialty examination of resident error disclosure and communication skills using simulation. J Grad Med Educ. 2018;10(4):438-441. doi:10.4300/JGME-D-17-00603.1

10. Krumwiede KH, Wagner JM, Kirk LM, et al. A team disclosure of error educational activity: Objective outcomes. J Am Geriatr Soc. 2019;67(6):1273-1277. doi:10.1111/jgs.15883

11. McDonough KA, White AA, Odegard PS, Shannon SE. Interprofessional error disclosure training for medical, nursing, pharmacy, dental, and physician assistant students. MedEdPORTAL. 21 Jul 2017;13:10606. doi:10.15766/mep_2374-8265.10606

12. Marshall C, Van Der Volgen J, Lombardo N, et al. A mixed methods approach to assess the impact of an interprofessional education medical error simulation. Am J Pharm Educ. 2020;84(2):7133. doi:10.5688/ajpe7133

13. Ragucci KR, Kern DH, Shrader SP. Evaluation of interprofessional team disclosure of a medical error to a simulated patient. Am J Pharm Educ. 2016;80(8):138. doi:10.5688/ajpe808138 
14. Poirier TI, Pailden J, Jhala R, Ronald K, Wilhelm M, Fan J. Student self-assessment and faculty assessment of performance in an interprofessional error disclosure simulation training program [published correction appears in Am J Pharm Educ. 2017 Aug;81(6):S7]. Am J Pharm Educ. 2017;81(3):54. doi:10.5688/ajpe81354

15. The National Telehealth Policy Resource Center. Center for Connected Health Policy. About Telehealth. What is telehealth? https://www.cchpca.org/what-is-telehealth/?category=live-video. Updated April 16, 2021. Accessed June 24, 2021.

16. Health Resources \& Services Administration. Telehealth programs. Available from https://www.hrsa.gov/ruralhealth/telehealth. Reviewed January 2021. Accessed June 24, 2021.

17. Totten AM, Hansen RN, Wagner J, et al. Telehealth for acute and chronic care consultations. comparative effectiveness review No. 216. (Prepared by Pacific Northwest Evidence-based Practice Center under Contract No. 290-2015-00009-I.) AHRQ Publication No. 19-EHC012-EF. Rockville, MD: Agency for Healthcare Research and Quality; April 2019. DOI: https://doi.org/10.23970/AHRQEPCCER216

18. Kalender-Rich J, Coffey C, Jernigan S, et al. Barney 3.0: A bicampus interprofessional hospital discharge simulation. J Am Geriatr Soc. 2018;67(S1):S75.

19. Lum E, van Galen LS, Car J. Competency-based training for entrustment in telehealth consultations. Pediatr Clin North Am. 2020;67(4):735-757. doi:10.1016/j.pcl.2020.04.013

20. Fischer SH, Uscher-Pines L, Roth E, Breslau J. The transition to telehealth during the first months of the COVID19 pandemic: Evidence from a national sample of patients. J Gen Intern Med. 2021;36(3):849-851. doi:10.1007/s11606-020-06358-0

21. Smith TS, Watts P, Moss JA. Using simulation to teach telehealth nursing competencies. J Nurs Educ. 2018;57(10):624-627. doi:10.3928/01484834-20180921-

Table 1. Median Survey Data Shared Question Pairs with Significance

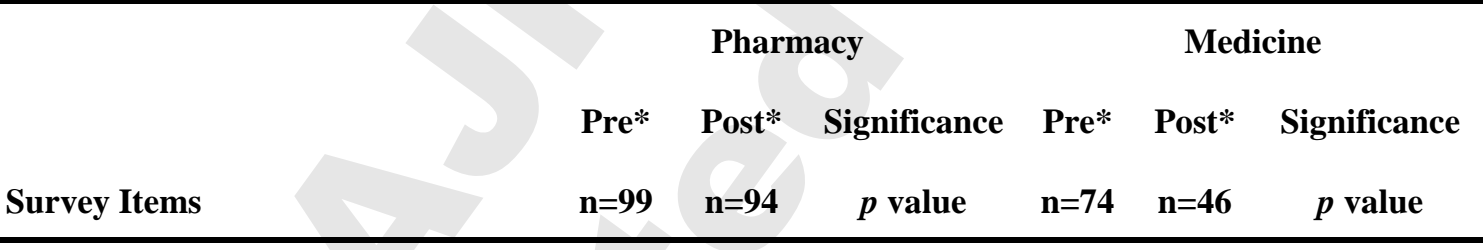

Telehealth services

I would use telemedicine consultative services again

\section{3}

I feel comfortable communicating with clinicians using a telemedicine system

Telemedicine is an acceptable way to receive consultative services

Pharmacist role

The primary role of community pharmacists is to fill and dispense prescription medications to patients

The community pharmacist is an important member of the interprofessional healthcare team

I feel confident that I know what MTM is when provided by a community pharmacist

Error identification and disclosure

I know how to identify how a medication error may have been caused

I am comfortable reporting to a fellow colleague/peer an

$\begin{array}{cccccc}4 & 4 & p<.001 & 3 & 4 & p<.001 \\ 4 & 4 & p<.001 & 3.5 & 4 & p<.001\end{array}$


intercepted or actual medication error I made

I am comfortable reporting to a fellow colleague/peer an

intercepted or actual medication error someone else made

\section{4}

4

$p<.001$

3

4

$p=.001$

*Responses based on the five-point Likert scale: $1=$ strongly disagree, $2=$ disagree, $3=$ neutral, $4=$ agree, $5=$ strongly agree.

MTM=Medication Therapy Management

Table 2. Assessment Rubric for Pharmacy Students

\section{Rubric Item}

$\begin{array}{cc}\text { Number }(\%) & \\ \text { of Students Completed } & \begin{array}{c}\text { Median } \\ (n=145)\end{array} \\ \text { Score }^{+}\end{array}$

Contributed to team discussion of error

Acknowledged error occurred

Positive Instance: Recognizes that an error has happened

$72(49.7)$

Presented error in a blame-free tone

Positive Instance: Talks about what happened without blaming others, responds

effectively to attempts to blame

$112(77.2)$

Build team consensus

Positive Instance: Solicits and respects team members' viewpoints about what happened; focuses on patient-centered goals, responds empathically to each other's distress about event, willing to speak against authority gradient

$130(89.7)$

Negotiated differences in opinion collaboratively

Positive Instance: Listens, paraphrases, yields floor/doesn't interrupt, summarizes to

check for mutual understanding

$129(89.0)$

Contributed to team planning for error disclosure

Advocates for full disclosure

Positive Instance: Lists reasons why full disclosure is desirable; Responds

respectfully and effectively to arguments presented to the contrary

$28(19.3)$

Plans role for disclosure

Positive Instance: Collaborates on a plan for the disclosure that includes who will

lead and what role each team member will play

$66(45.4)$

Anticipates patient's questions and reactions

Positive Instance: Anticipates likely questions and reactions from patient

Plans response to patient

Positive Instance: Formulates reasonable responses to patient from team

$30(20.7)$

0

Communication

Appropriately uses medical jargon, minimizes pharmacy specific jargon

$142(97.9)$

Uses appropriate communication to suit the team members

Has an organized approach to provider encounter

$112(77.2)$

Pace, volume, and eye contact are appropriate to communicate clearly

$137(94.5)$

Overall rubric median completion score

$+0=$ non-completion of item by $>50 \%$ of students, $1=$ completion of item by $>50 \%$ of students 


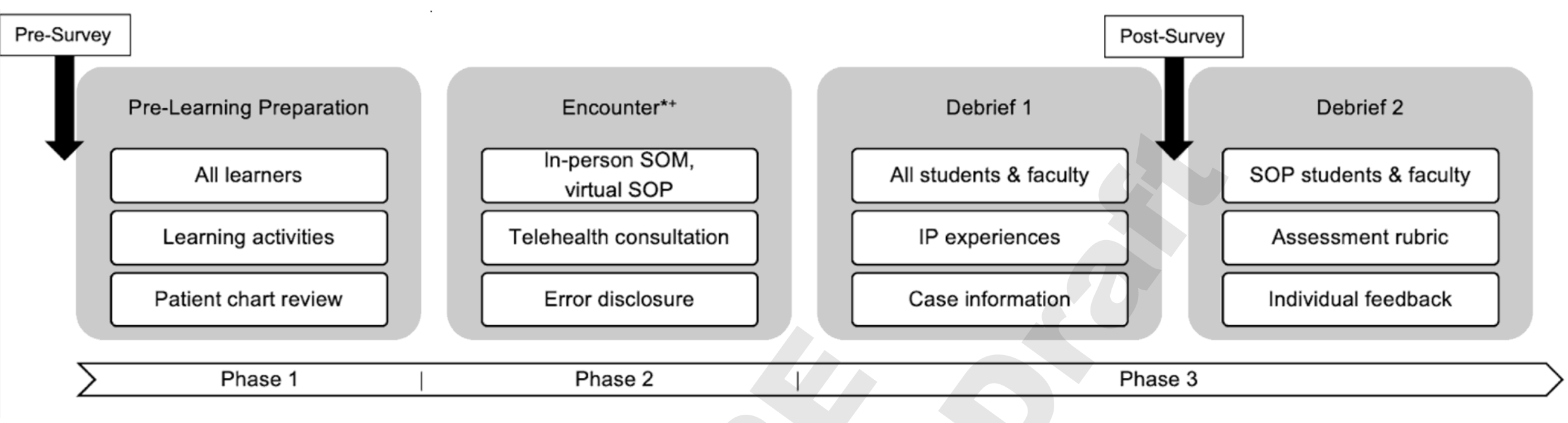

This simulation included three phases of student engagement. Phase one included individual student preparation for the simulation. All students reviewed pertinent patient chart information and a medication error identification and disclosure podcast. Additionally, pharmacy students viewed a MTM encounter video and medical students participated in a discharge planning simulation. Phase two included the telehealth consultation between medicine and pharmacy students for the error disclosure. The final phase included faculty led interprofessional and profession-specific debrief sessions.

Key: *=real-time video viewing, +=video recorded for later review, IP=interprofessional, SOM=school of medicine, SOP=school of pharmacy 